\title{
INFLUENCE OF VARYING TUNE WIDTH ON THE ROBUSTNESS OF THE LHC TUNE PLL AND ITS APPLICATION FOR CONTINUOUS CHROMATICITY MEASUREMENT.
}

\author{
R.J. Steinhagen, A. Boccardi, M. Gasior, O.R. Jones, K.K. Kasinski \\ CERN, Geneva, Switzerland
}

\begin{abstract}
Tune and chromaticity measurement is an integral part of safe and reliable LHC operation. Tight tolerances on the maximum transverse beam excursions allow oscillation amplitudes of less than $30 \mathrm{ìm}$. This leaves only a small margin for transverse beam and momentum excitations required for measuring tune and chromaticity. This contribution discusses a robust tune phase-locked-loop (PLL) operation in the presence of non-linearities and varying chromaticity. The loop design was tested at the SPS, using the LHC PLL prototype system. The system was also used to continuously measure tune width and chromaticity, using resonant transverse excitations of the tune side-slopes.
\end{abstract}

CERN, 


\title{
INFLUENCE OF VARYING TUNE WIDTH ON THE ROBUSTNESS OF THE LHC TUNE PLL AND ITS APPLICATION FOR CONTINUOUS CHROMATICITY MEASUREMENT
}

\author{
R.J. Steinhagen, A. Boccardi, M. Gasior, O.R. Jones, K.K. Kasinski (CERN, Geneva, Switzerland)
}

\section{Abstract}

Tune and chromaticity measurement is an integral part of safe and reliable LHC operation. Tight tolerances on the maximum transverse beam excursions allow oscillation amplitudes of less than $30 \mu \mathrm{m}$. This leaves only a small margin for transverse beam and momentum excitations required for measuring tune and chromaticity. This contribution discusses a robust tune phase-locked-loop (PLL) operation in the presence of non-linearities and varying chromaticity. The loop design was tested at the SPS, using the LHC PLL prototype system. The system was also used to continuously measure tune width and chromaticity, using resonant transverse excitations of the tune side-slopes.

\section{INTRODUCTION}

Recent improvements in hadron colliders have led to significantly increased stored beam energies which require excellent control of particle loss inside a superconducting machine. Thus, most requirements on key beam parameters in superconducting hadron colliders strongly depend on the capability to control particle losses inside the accelerator. In the case of the Large Hadron Collider (LHC), the Cleaning System has the tightest constraints on beam position stability of better than $25 \mu \mathrm{m}$ during nominal operation at the location of the collimator jaws. The horizontal dispersion relates this to an upper limit for momentum modulation of about $\frac{\Delta p}{p}<10^{-4}$. Both constraints leave only a small margin for beam excitation required for the measurement and control of tune and chromaticity [1].

Table 1 summarises the beam stability requirements for different stages of operation and expected (maximum) ramp-induced perturbations [3].

\section{LHC TUNE MEASUREMENT SYSTEMS}

Nominal LHC Cleaning System operation allows transverse oscillations of only a few ten micrometres before the beam halo is pushed and eventually lost in the collimators. This limits classic tune techniques relying on turn-by-turn beam position monitor measurements in response to large kicks in the few 100 micro- to millimetre range. In response to these limitations, a robust diode-based base-band tune meter (BBQ) has been developed at CERN, which is capable of detecting small transverse oscillations in the nanometre range [4]. The BBQ is the baseline tune meter for both passive monitoring of residual oscillation as well as in combination with kicked, chirped ( [5]) or continuous beam excitation such as the Phase-Locked-Loop (PLL). 06 Instrumentation, Controls, Feedback \& Operational Aspects
While techniques using 'kicked' and 'chirped' excitations are limited by the finite excitation length and Landau decoherence, driven coherent detection of the tune beam response using PLLs have typically higher noise rejection, and usually allow much longer acquisition and integration periods. In addition, a resonant Schottky pickup system has been developed and will further complement the BBQbased systems during operation at top energy [6].

\section{PHASE-LOCKED-LOOP SCHEMATIC}

As working principle, PLL control systems continuously adjust phase $\varphi$ and frequency $f_{e}$ of their reference exciter to match and track changes of the betatron tunes. A classic application of PLL is to measure the chromaticity by modulating the beam momentum using the RF frequency while tracking the tune. The modulation amplitude is then proportional to the linear machine chromaticity while the barycentre is a measure of the unperturbed tunes.

In good approximation, the tune resonance can be described by a second-order harmonic oscillator. Thus the tune resonance is found once the phase between excited and measured oscillation equals $\pi / 2$. The corresponding LHC PLL block diagram is shown in Figure 1.

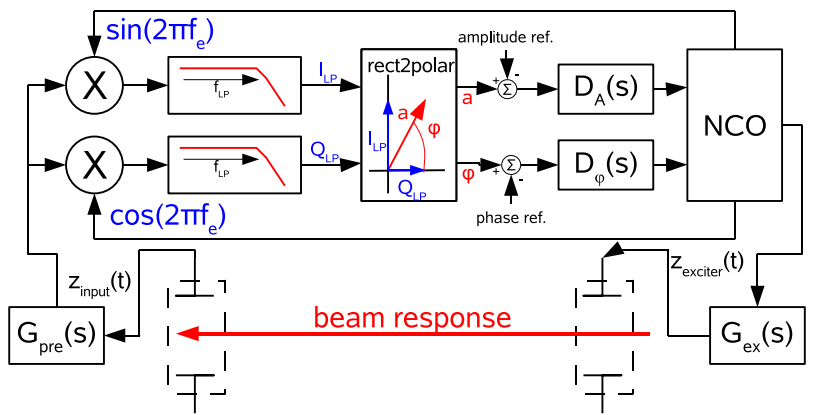

Figure 1: LHC Tune Phase-Locked-Loop Schematics.

The system mixes the digitised beam signal with the sine and cosine components of the excitation signal and uses low-pass filters in order to remove the $2 \pi f_{e}$ frequency component that is created in the mixing process. The resulting signals are passed through a rectangular-to-polar converter that separates signal phase and amplitude which can then further be treated by two independent controllers. The actual control input $\Delta \varphi$ is shifted by $\pi / 2$ to zero in order to obtain a bipolar signal around the tune resonance. Negative phases thus indicate that the excitation frequency is below the tune resonance, and positive phases the opposite. The control input is further compensated for other non-beam related contributions to the measured phase shift such as constant lag due to data processing, cables transmission delays,

T05 Beam Feedback Systems 
Table 1: Beam stability requirements in relation to expected (brackets: worst case) ramp-induced perturbations [3].

\begin{tabular}{|l|c|c|c|c|c|}
\hline & Orbit & Tune & Chromaticity & Energy & Coupling \\
\cline { 2 - 6 } & {$[\sigma]$} & {$\left[f_{r e v}\right]$} & {$\left[Q^{\prime}\right]$} & {$[|\Delta p / p|]$} & {$\left[C^{-}\right]$} \\
\hline Pilot: & - & \pm 0.1 & +10 & - & 0.1 \\
\hline Commissioning: & \pm 1 & \pm 0.015 & $>0 \& \pm 5$ & $<10^{-4}$ & $\ll 0.1 \rightarrow 0.003$ \\
\hline Nominal: & \pm 0.3 & $\pm 0.003 / \pm 0.001$ & $2 \pm 1$ & $<10^{-4}$ & $\ll 0.001$ \\
\hline \hline Perturbations: & $0.5(30 \mathrm{~mm})$ & $0.14(0.18)$ & $70(300)$ & $\pm 1.5 \cdot 10^{-4}$ & $0.01(0.1)$ \\
\hline Drift Rates: & $<25 \mu \mathrm{m} / \mathrm{s}$ & $\ll 10^{-3} \mathrm{~s}^{-1}$ & $<1.3 \mathrm{~s}^{-1}$ & $\ll 10^{-5} \mathrm{~s}^{-1}$ & - \\
\hline
\end{tabular}

analogue pre-filters suppressing dominant harmonics (e.g. revolution frequency) and the response of the beam exciter itself. The LHC tune PLL system is implemented on the same FPGA-based data acquisition board as the continuous FFT processing chain, providing operational flexibility and redundancy in case of hardware failure [5].

Providing operation at or close to the phase-lock condition, the phase control loop dynamics can be linearised and reduced to a first-order system with the open loop gain $K_{0}$ given by the slope of the phase response at the location of the tune, and the inverse time constant $\tau$ by the bandwidth of the low-pass filter. The LHC PLL controller design has been based on Youla's affine parameterisation, an optimal control approach, and yields a simple proportional-integral controller [7]:

$$
D_{\varphi}(s)=K_{p}+K_{i} \frac{1}{s} \text { with } K_{p}=K_{0} \frac{\tau}{\alpha}, K_{i}=K_{0} \frac{1}{\alpha}
$$

Proportional $K_{p}$ and integral gain $K_{i}$ are coupled. The inverse of the free parameter $\alpha$ defines the effective closedloop bandwidth. Depending on the operational scenario ('gain-scheduling'), the single parameter $\alpha$ thus facilitates the trade-off between a fast tracking PLL $\left(f_{b w} \approx 6 \mathrm{~Hz}\right)$ but with larger measurement resolution $\left(\Delta Q_{\text {res }} \approx 10^{-3}\right)$ and a PLL having smaller measurement resolution $\left(\Delta Q_{\text {res }} \approx\right.$ $10^{-4} \ldots 10^{-6}$ ) but also smaller tracking bandwidth.

Figure 2 shows a typical PLL-based tune trace measured in the SPS using a LHC type beam that accelerated from $26 \mathrm{GeV} / \mathrm{c}$ (time: $0 \mathrm{~s}$ ) to a final momentum of $450 \mathrm{GeV} / \mathrm{c}$ $(6.5 \mathrm{~s})$ and resonantly extracted using the lattice sextupoles. The tune trace (blue), residual phase error (red), and amplitude signal (green) are shown. The initial fractional tune $q_{h} \approx 0.76$ is quickly changed below (6.5-7.5 s) and slowly brought towards the third order resonance $\left(q_{h}=0.66\right)$ before the beam is eventually fully extracted and the beam signal lost $(\approx 8.3 \mathrm{~s})$. The less than $\pi / 2$ phase error and

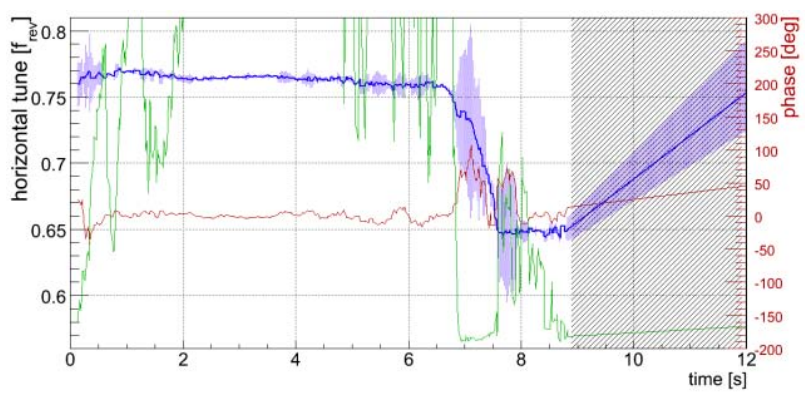

Figure 2: LHC PLL tune tracking example in the SPS.

the non-vanishing amplitude response indicate that the PLL was capable of tracking tune changes of up $\Delta Q \approx 0.1$ within about 200 to 300 milliseconds which is orders of 06 Instrumentation, Controls, Feedback \& Operational Aspects magnitude faster than the expected tune changes and requirements in the LHC summarised in Table 1.

\section{PLL ROBUSTNESS}

The robustness of the tune PLL is defined by the ability to track the unperturbed betatron tunes in the presence of discrepancies between the actual and mathematical process model that has been used to design the feedback controller as well as the impact of noise and external disturbances on the specified tracking performance. The presence of strong betatron coupling, coupled bunch instabilities or strong resonances other than the tune may potentially perturb or even compromise the PLL function if not compensated or included into the loop [7].

\section{Dependence on Tune Width}

As described above, most classic tune PLL implementations assume that the open loop gain $K_{0}$ which depends on the phase slope at the location of the tune resonance is constant. Due to varying chromaticity, amplitude detuning, beam-beam, electron cloud, impedance and other effects, the tune width, thus the phase slope and $K_{0}$ may change. Using linear control design only, this cross-dependence implies either a controller design that is optimal for large tune widths, which becomes sensitive to noise and potentially instable for small tune widths, or a controller design that is optimal for small tune width but lags behind the real tune for large width.

Measurements performed in the SPS suggests that the tune width measured by above BBQ-based LHC PLL appears to be linearly dependent on the chromaticity $Q$ '. In order to investigate and assess this dependency, the beam has been excited at two additional frequencies in the vicinity of the primary exciter used for tracking the tune. While the phase detection is the same as for the central exciter, their frequencies are kept constant with respect to the central exciter. In order to obtain a better phase measurement resolution and to minimise the spectral leakage between the side- and central exciters, the side-exciters' phase signals were subjected to a tighter low-pass filtering $\left(f_{L P} \approx\right.$ $0.1 \mathrm{~Hz}$ ). Figure 3 shows a typical beam response function measurement performed in the SPS. The lower (red vertical line) and upper (blue vertical line) side exciters are indicated around the tune (dashed black line). The sideexciter frequencies were set to be $\Delta Q_{\text {sex }} \approx 5 \cdot 10^{-4}$ ) apart from the tune. The beam transfer function was scanned within about 30 seconds corresponding to a scanning speed of about $\Delta Q / \Delta t \approx 3 \cdot 10^{-4} \mathrm{~s}^{-1}$. The resonance at 0.192 


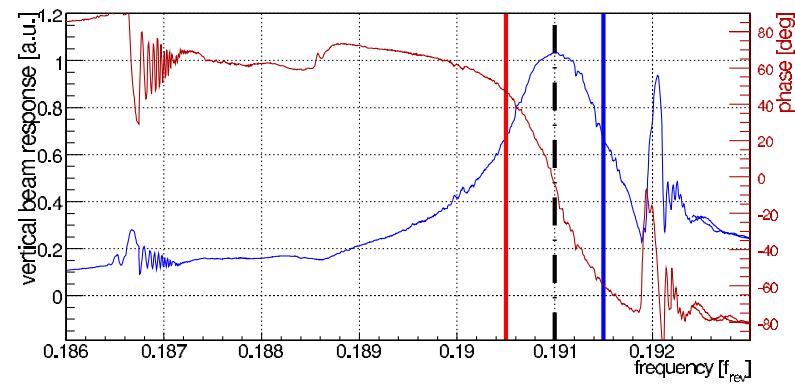

Figure 3: SPS driven beam response example.

was observed for large chromaticities $\left(Q^{\prime} \approx 10\right)$ seemingly being a synchrotron side-band of the betatron tune $\left(Q_{s} \approx 0.001\right)$. However the corresponding lower sideband of the tune resonance is missing. Further, though the PLL resolution was sufficient, the side-bands around the tune at twice and thrice the synchrotron frequency were not visible either. This indicates that the classic theory in its simple form predicting symmetric synchrotron side-bands around the tune for bunched beams does not seem to apply for this type of measurement.

In the given tune resonance approximation, the measured un-normalised side-exciter phase and tune width $\Delta Q$ are related through

$$
\tan (\varphi)=\frac{\Delta Q \cdot Q_{0} Q_{\text {sex }}}{Q_{\text {sex }}^{2}-Q_{0}^{2}}
$$

with $Q_{0}$ being the tune and $Q_{\text {sex }}$ the side exciter frequency normalised by the revolution frequency. In order to obtain similar chromaticity values, the tune width has been normalised by the average r.m.s. bunch momentum spread similar to the un-bunched beam case. Using the same formalism, it can be shown that the inverse amplitude response at the resonance frequency is also proportional to the tune width. Figure 4 shows the combined results of the tune width based chromaticity measurement obtained using the phase of the lower (red) and upper (blue) side-exciters. In addition, the inverse amplitude response of the central exciter (black) has been superimposed. Since the amplitude response directly depends on the excitation strength, tunewidth based chromaticity estimated using the inverse amplitude response was scaled by a constant factor and shifted to match the phase-based one. The visible steps correspond to relative increases of $\Delta Q^{\prime}=2.6$ units of the true machine chromaticity. The orbit was not perfectly centred inside all lattice sextupoles, and thus each chromaticity change also induced a small tune change that caused the visible transients due to the lag of the tune PLL. Though the mea-

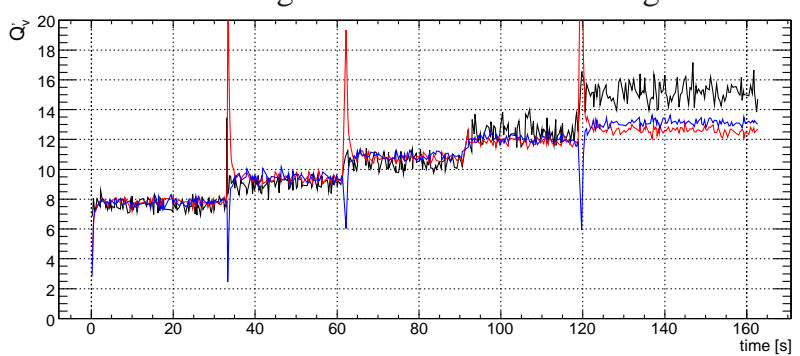

Figure 4: LHC PLL $\Delta Q$ tracking example in the SPS. sured property changed linearly with chromaticity, the absolute measured chromaticity property showed a constant offset that needed to be calibrated with respect to a classical momentum-modulation-based chromaticity measurement. This type of measurement was reproducible for various bunch intensities and patterns in the SPS. Though strictly measuring the tune width, the property could be used for relative chromaticity or tune width change measurements independent of the constant offset uncertainty and thus potentially exploited as a chromaticity tracker within the LHC chromaticity feedback loop that needs to stabilise $Q$, around a given reference point rather than measuring the absolute value.

Though the advantage of this measurement method is that it requires only small transverse excitations and no further momentum modulations, there still remain uncertainties such as dependence on non-linearities due to higherorder multipole fields, impedance and longitudinal RF properties as well as the underlying mathematical model that needs further assessment prior to a deployment as a robust chromaticity tracker. Thus for the time being, the baseline will still be based on the classic momentummodulation based chromaticity measurement.

\section{CONCLUSIONS}

The prototype tests of the BBQ-based tune PLL showed good results in the SPS. Its range covers two extreme modes of operation: the ability to track fastest tune changes of up to $\Delta Q \approx 0.1$ within 200 to $300 \mathrm{~ms}$ or to achieve tune measurement resolutions down to $\Delta Q_{\text {res }} \approx$ $10^{-4} \ldots 10^{-6}$, although with reduced bandwidth. The controller design choice reduces the number of free parameters to the effective closed loop bandwidth that can be used to optimise the loop in between the 'fast tracking' and 'precise tracking' mode of operation.

The same system was used to measure tune width. These side-exciter based measurements in the SPS indicated that the measured tune width is linearly dependent on chromaticity, which potentially could be exploited for future LHC chromaticity feedback loops. However, the underlying systematic of the measurement, in particular the dependence on non-linear effects requires further study.

\section{REFERENCES}

[1] R. Assmann, CERN, Chamonix XII, 2003

[2] S. Fartoukh, O. Brüning, CERN-LHC-Project-Report-501

[3] R.J. Steinhagen, CERN, Chamonix XV, 2006

[4] M. Gasior, O.R. Jones, CERN-LHC-Project-Report-853

[5] A. Boccardi et al., "The FPGA-based Continuous FFT Tune Measurement System for the LHC and its Tests at the CERN SPS", these proceedings

[6] F. Caspers et. al., "The 4.8 GHz LHC Schottky Pick-up System", these proceedings

[7] R.J. Steinhagen, "Feedbacks on Tune and Chromaticity", DIPAC'07 